\title{
Effect of thermomechanical treatment on the microstructure and mechanical properties of a novel heavily alloyed nickel base superalloy
}

\author{
V. M. Imayev ${ }^{\dagger, 1}$, Sh. Kh. Mukhtarov ${ }^{1}$, A. V. Logunov², A. A. Ganeev ${ }^{1}$, \\ R. V. Shakhov' ${ }^{1}$ R. M. Imayev ${ }^{1}$ \\ †vimayev@mail.ru
}

${ }^{1}$ Institute for Metals Superplasticity Problems of RAS, 39 Khalturina St., Ufa, 450001, Russia ${ }^{2}$ PJSC “UEC-Saturn”, 163 Lenina Av., Rybinsk, 152903, Russia

The work has been devoted to study of microstructure and mechanical properties of a novel heavily alloyed heat resistant nickel base superalloy in the cast and heat treated condition and the thermomechanically treated (TMT) condition. The cast condition after heat treatment including homogenization annealing, solid solution treatment and ageing was characterized by a coarse $\gamma$ grain size and uniformly distributed dispersed $\gamma^{\prime}$ precipitates with a size of $d_{\gamma}=0.1-0.25 \mu \mathrm{m}$. It was established that TMT under optimal conditions led to development of recrystallization processes, which provided formation of refined recrystallized microstructure with a $\gamma$ grain size of $d_{\gamma}=2-60 \mu \mathrm{m}$. The obtained microstructure condition contained mostly dispersed $\gamma^{\prime}$ precipitates with a size of $d_{\gamma}=0.1-0.3 \mu \mathrm{m}$. The volume fraction of relatively coarse $\gamma^{\prime}$ particles, which were not dissolved during TMT, was about 10\%. The TMT condition was aged in the same manner as the cast condition. Tensile tests and long-term strength tests were performed for the both superalloy conditions. The strength properties in the TMT condition were found by $30-50 \%$ higher than those obtained in the cast and heat treated condition. At the same time, the ductility in the TMT condition was found also significantly higher than in the cast and heat treated condition. The following tensile properties were obtained for instance at room temperature: $\sigma_{\mathrm{UTS}} / \sigma_{0.2}=1802 / 1355 \mathrm{MPa}$ and $\delta=16 \%$ for the TMT and aged condition, and $\sigma_{\mathrm{UTS}} / \sigma_{0.2}=1255 / 1132 \mathrm{MPa}$ and $\delta=8.5 \%$ for the cast and heat treated condition. The long-term strength at $650^{\circ} \mathrm{C}$ in the TMT and aged condition was found also to be significantly higher than that in the cast and heat treated condition. Excellent mechanical properties of the superalloy after TMT are explained in terms of the increased amount of the $\gamma$ ' phase, the effective solid solution strengthening due to heavy alloying with refractory metals and the refined microstructure obtained after TMT.

Keywords: heat resistant nickel base superalloy, thermomechanical treatment, microstructure, mechanical properties.

УДК: 669.295

\section{Влияние деформационно-термической обработки на микроструктуру и механические свойства нового высоколегированного никелевого сплава}

\author{
Имаев В.М. ${ }^{\dagger, 1}$, Мухтаров Ш.Х. ${ }^{1}$, Логунов А. В. ${ }^{2}$, Ганеев А. А. ${ }^{1}$, Шахов Р. В. ${ }^{1}$, Имаев Р. М. ${ }^{1}$ \\ ${ }^{1}$ Институт проблем сверхпластичности металлов РАН, ул. Халтурина, 39, Уфа, 450001, Россия \\ ${ }^{2}$ ПАО «ОДК-Сатурн», пр. Ленина, 163, Рыбинск, 152903, Россия
}

Работа посвящена исследованию микроструктуры и механических свойств нового высоколегированного жаропрочного никелевого сплава в литом состоянии, подвергнутом только термической обработке, и состоянии после деформационно-термической обработки (ДТО). Литое состояние после термической обработки, включавшей в себя гомогенизационный отжиг, обработку на твердый раствор и заключительное старение, характеризовалось крупным размером $\gamma$ зерен и равномерно распределенной дисперсной упрочняющей $\gamma^{\prime}$ фазой размером $d_{\gamma^{\prime}}=0.1-0.25$ мкм. Установлено, что ДТО существенно преобразует микроструктуру за счет однородного развития рекристаллиза- 
ционных процессов, обеспечивающих формирование в сплаве структуры с размером рекристаллизованных $\gamma$ зерен в диапазоне $d_{\gamma}=2-60$ мкм. Полученное состояние содержало в основном дисперсную $\gamma^{\prime}$ фазу размером $d_{\gamma^{\prime}}=0.1-0.3$ мкм. Содержание относительно крупной $\gamma^{\prime}$ фазы, не растворившейся при ДТО, составляло около 10 об.\%. Деформированное состояние было подвергнуто старению в тех же условиях, что и в случае литого состояния. Были выполнены механические испытания полученных состояний сплава на растяжение и длительную прочность. Состояние после ДТО показало на 30 - 50\% более высокую прочность при комнатной и повышенной температурах, чем литое состояние, подвергнутое только термической обработке. При этом пластичность после ДТО оказалась значительно выше. Так, при комнатной температуре было получено $\sigma_{\mathrm{B}} / \sigma_{0.2}=1802 / 1355$ МПа и $\delta=16 \%$ в состоянии после ДТО и старения, и $\sigma_{\mathrm{B}} / \sigma_{0.2}=1255 / 1132$ МПа и $\delta=8.5 \%$ в литом состоянии, подвергнутом только термической обработке. Длительная прочность при $650^{\circ} \mathrm{C}$ также оказалась значительно выше в состоянии после ДТО и старения. Превосходные механические свойства сплава после ДТО объясняются, прежде всего, повышенным содержанием $\gamma^{\prime}$ фазы, эффективным твердорастворным упрочнением благодаря высокому легированию тугоплавкими элементами и измельчением микроструктуры в результате ДТО.

Ключевые слова: жаропрочный сплав на основе никеля, деформационно-термическая обработка, микроструктура, механические свойства.

\section{1. Введение}

Жаропрочные никелевые сплавы широко применяются в газотурбинных двигателях (ГТД) в качестве конструкционных материалов для деталей ответственного назначения, таких как диски [1-3]. Для повышения экономичности и эффективности ГТД стремятся повысить температуру эксплуатации и нагруженность дисков, что стимулирует разработку более прочных и жаропрочных никелевых сплавов. В отношении поликристаллических никелевых сплавов, являющихся наиболее конкурентоспособными с точки зрения стоимости, речь обычно идет о повышении легирования элементами, увеличивающими содержание упрочняющей $\gamma^{\prime}$ фазы и температуры ее полного растворения, а также обеспечивающими эффективное твердорастворное упрочнение [1-5]. Однако высоколегированные никелевые сплавы в литом состоянии являются чрезвычайно труднодеформируемыми. Неоднородная гетерогенная структура и сильная дендритная ликвация в слитке ограничивают деформируемость сплава даже при температурах близких к температуре полного растворения $\gamma^{\prime}$ фазы. В то же время достижение высоких механических свойств в никелевых сплавах, изготовленных литьем, невозможно без трансформации крупнозернистой литой структуры в однородную рекристаллизованную структуру. Для повышения деформируемости высоколегированного никелевого сплава в литом состоянии его подвергают предварительному гомо- и гетерогенизационному отжигу для уменьшения дендритной ликвации и гетерогенности структуры $[1,3]$, а затем подбирают соответствующие схему и температурно-скоростные условия деформационной обработки. Для однородного развития рекристаллизационных процессов и формирования мелкозернистой структуры в труднодеформируемых материалах, подобных никелевым сплавам, наиболее благоприятными с точки зрения обработки металлов давлением являются схемы, обеспечивающие всестороннеесжатие, которое реализуется, например, пригидроэкструзии, сжатии в толстостенной оболочке и др.

В настоящей работе объектом исследования явился высоколегированный никелевый сплав СДЖС-15, разработанный в ПАО «ОДК-Сатурн» (г. Рыбинск) с помощью компьютерного проектирования и предполагаемый для применения в качестве материала дисков для ГТД с рабочей температурой до $850^{\circ} \mathrm{C}$. Сплав содержит повышенное количество $\gamma^{\prime}$-образующих легирующих элементов ( $\mathrm{Ti}, \mathrm{Al}, \mathrm{Nb}, \mathrm{Ta})$, а также элементов, обеспечивающих твердорастворное упрочнение (Co, Re, Mo, W). По своему составу сплав СДЖС-15 близок к некоторым монокристаллическим никелевым сплавам.

Целью работы явилось сравнительное исследование микроструктуры и механических свойств сплава в литом состоянии, подвергнутом только термической обработке (ТО), и состоянии после деформационно-термической обработки (ДТО). Схема деформации и температурноскоростные условия ДТО были разработаны ранее $[7,8]$.

\section{2. Материал и методики эксперимента}

Номинальный состав сплава СДЖС-15: Ni-28(Cr, Co)12.5(Al, Ti, Nb, Ta)-9(Mo, W, Re)-0.17(C, La, Y, Ce, B) (вес.\%). Слитки сплава $\varnothing 100 \times 180$ мм были изготовлены в НТЦ «Технологии специальной металлургии» (МИСиС, Москва). Реальные составы слитков соответствовали номинальному составу сплава.

Температуру полного растворения $\gamma^{\prime}$ фазы $\left(T_{s}\right)$ определяли методом пробных закалок от температур вблизи температуры полного растворения $\gamma^{\prime}$ фазы. Она составила $T_{s} \approx 1220^{\circ} \mathrm{C}$. Для оценки механических свойств исходного сплава литое состояние сплава подвергали ТО, состоящей из гомогенизационного отжига в диапазоне температур $\left(T_{s}-120\right) \div T_{s}$, последующей обработки на твердый раствор с охлаждением на воздухе и старения. Из термически обработанной заготовки вырезали образцы для механических испытаний.

Перед проведением ДТО литое состояние сплава подвергали гомо- и гетерогенизационному отжигу в диапазоне температур $\left(T_{s}-120\right) \div\left(T_{s}+20\right)$, затем термообработанную заготовку сплава помещали в специальный контейнер из нержавеющей стали и деформировали в одном направлении сжатием в два этапа в квазиизотермических условиях спромежуточным рекристаллизационным отжигом. Температура нагрева заготовки в контейнере составляла $\left(T_{s}-45\right)^{\circ} \mathrm{C}$, темпе- 
ратура на бойках $-930^{\circ} \mathrm{C}$, деформацию осуществляли со скоростью $\varepsilon^{\prime} \approx 10^{-2} \mathrm{c}^{-1}$, общая степень деформации составила $e \approx 1.4$ [8]. Деформированные заготовки охлаждали на воздухе и подвергали старению, после чего из них были вырезаны образцы для механических испытаний. Старение после ТО и ДТО проводили в идентичных условиях: при $T=860^{\circ} \mathrm{C}$ (6 ч.) и $T=750^{\circ} \mathrm{C}$ (32 ч.) с последующим охлаждением на воздухе.

Механические испытания были выполнены на растяжение при температурах $20-850^{\circ} \mathrm{C}$ и на длительную прочность при $650^{\circ} \mathrm{C}$ в течение 100 часов. Для кратковременных испытаний использовали плоские образцы с размерами рабочей части $10 \times 3 \times 2$ мм $^{3}$, для длительных испытаний - с размерами рабочей части $17 \times 3 \times 3$ мм $^{3}$. Испытания были выполнены на воздухе.

Микроструктурные исследования в случае деформированных заготовок проводили с центральной части деформированных образцов. Для микроструктурных исследований использовали сканирующую электронную микроскопию (СЭМ) в режиме обратно-рассеянных электронов (BSE). Энергодисперсионная приставка микроскопа была использована при проведении энергодисперсионного микрорентгеноспектрального (EDX) анализа. Для состояния, подвергнутого ДТО, был выполнен анализ с помощью дифракции обратнорассеянных электронов (EBSD анализ) с шагом сканирования 1 мкм. EBSD анализ был выполнен с исполь- зованием программного обеспечения CHANNEL 5. Границы зерен с разориентировкой менее $2^{\circ}$ были исключены из рассмотрения, принимая во внимание точность измерения. Высокоугловыми границами зерен считали границы зерен с углом разориентировки более $15^{\circ}$. Перед изучением микроструктуры поверхность образцов подвергали механической и электролитической полировке.

\section{3. Результаты и обсуждение}

\section{1. Микроструктура сплава}

На Рис. 1a,b представлена микроструктура сплава в исходном литом состоянии. Была получена типичная для никелевых сплавов крупнозернистая дендритная структура. Выделения $\gamma^{\prime}$ фазы имели размер преимущественно 0.2-0.5 мкм; кроме того, в микроструктуре присутствовали относительно крупные выделения $\gamma^{\prime}$ фазы размером 1-2 мкм (Рис. 1b). Относительно крупный размер $\gamma^{\prime}$ фазы объясняется медленной скоростью охлаждения слитка. Объемная доля $\gamma^{\prime}$ фазы составляла около $68 \%$ [7,8]. Светлые карбидные частицы равноосной и вытянутой формы имели размер $0.5-20$ мкм.

На Рис. 1c, d представлена микроструктура сплава после ТО. Благодаря ТО уровень дендритной

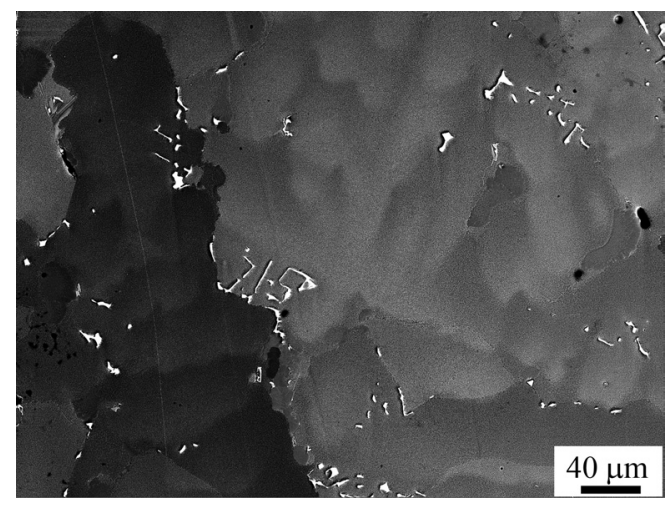

a

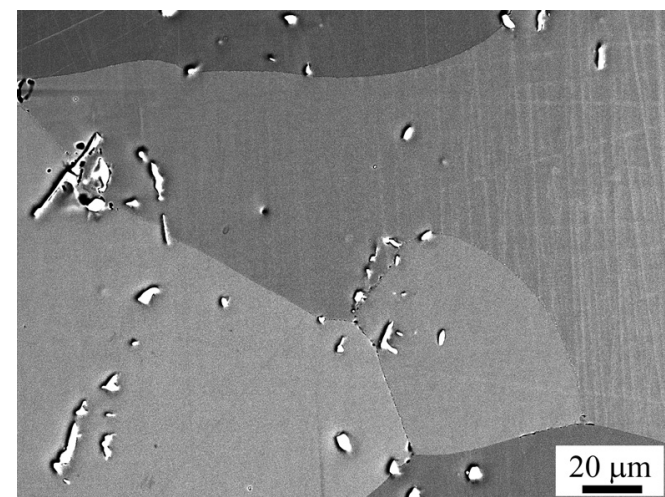

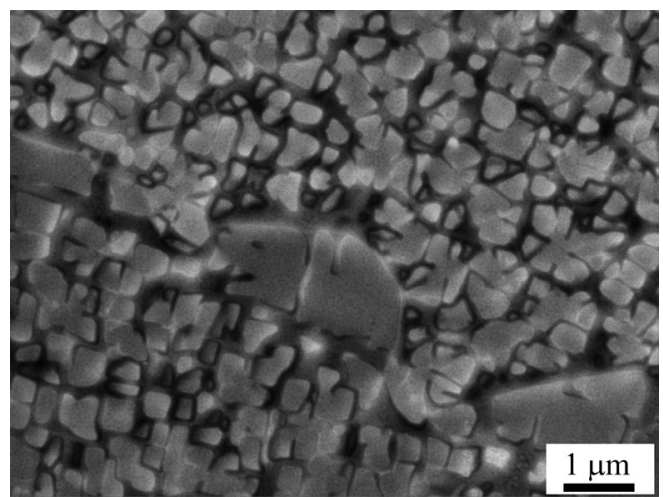

b

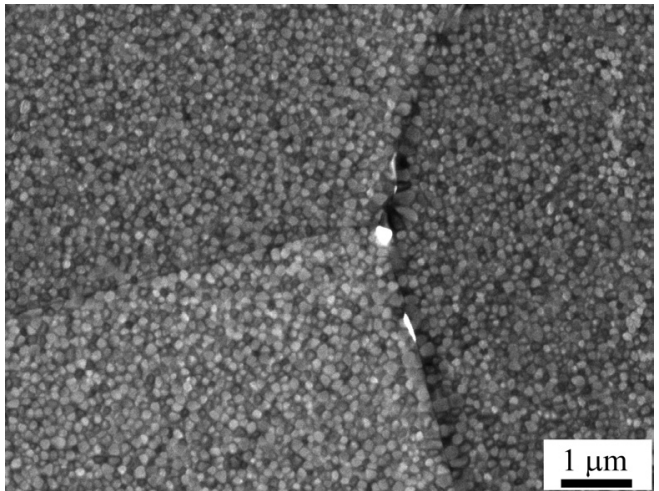

Рис. 1.Электронно-микроскопические изображения микроструктуры сплава СДЖС-15: в исходном литом состоянии (a, b), в литом состоянии, подвергнутом термической обработке (c, d); размер $\gamma^{\prime}$ выделений $(\mathrm{b}, \mathrm{d})$ сильно различался до и после термической обработки (СЭМ, ВSE).

Fig. 1. Electron microscope images of the SDZhS-15 superalloy: in the initial as-cast condition (a, b), in the cast and heat treated condition $(c, d)$; the $\gamma^{\prime}$ precipitates had different sizes (b, d) before and after heat treatment (SEM, BSE). 
ликвации в сплаве уменьшился, области химических неоднородностей не наблюдались. Размер $\gamma$ зерен составил 100...500 мкм. Дисперсная $\gamma^{\prime}$ фаза, однородно распределенная в объеме материала и имеющая округлую морфологию, имела размер 0.1-0.25 мкм (Рис. 1d). В отличие от исходного литого состояния, относительно крупные частицы $\gamma^{\prime}$ фазы размером 1-2 мкм не наблюдались, что связано с почти полным растворением $\gamma^{\prime}$ фазы при обработке на твердый раствор и выделением дисперсной $\gamma^{\prime}$ фазы при охлаждении на воздухе и старении. Объемная доля $\gamma^{\prime}$ фазы после ТО не изменилась. В микроструктуре, как и в исходном литом состоянии, также наблюдались светлые карбидные частицы равноосной и вытянутой формы.

На Рис. 2 представлена ориентационная карта и спектр разориентировок границ зерен, полученные с помощью EBSD анализа с центральной части заготовки сплава, подвергнутой ДТО. Видно, что была достигнута полностью рекристаллизованная микроструктура с преимущественно высокоугловыми границами зерен. Как было показано ранее, основным процессом в ходе выполненной ДТО является непрерывная динамическая рекристаллизация [8]. Размер рекристаллизованных $\gamma$ зерен варьировался в диапазоне $d=2-60$ мкм (Рис. 2 a). Следует отметить, что преимущественно рекристаллизованная микроструктура была достигнута во всем объеме деформированной заготовки сплава благодаря деформации в контейнере.

Микроструктура сплава после ДТО содержала в основном дисперсные выделения $\gamma^{\prime}$ фазы размером 0.1-0.3 мкм и небольшое количество, около 10 об.\%, относительно крупных выделений $\gamma^{\prime}$ фазы размером 1-3 мкм (Рис. 3). Крупные выделения $\gamma^{\prime}$ фазы не были растворены при температуре деформации, в то время как дисперсные частицы выделились при охлаждении деформированной заготовки на воздухе. Дисперсные выделения $\gamma^{\prime}$ фазы имели округлую морфологию. Объемная доля $\gamma^{\prime}$ фазы после ДТО в сравнении

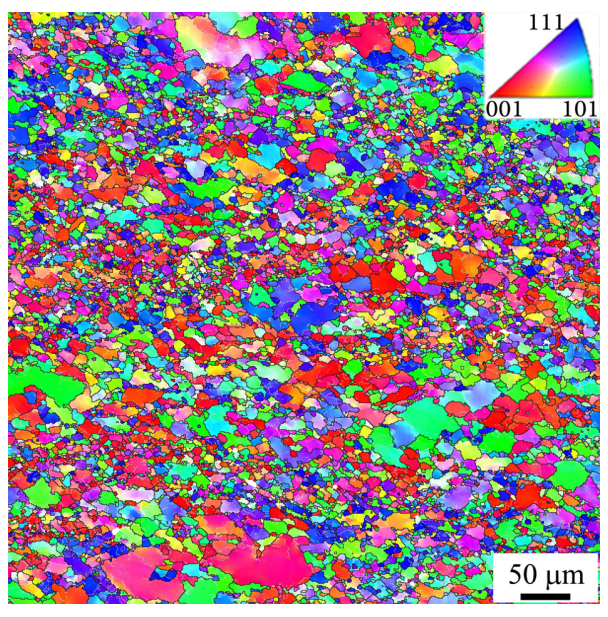

a

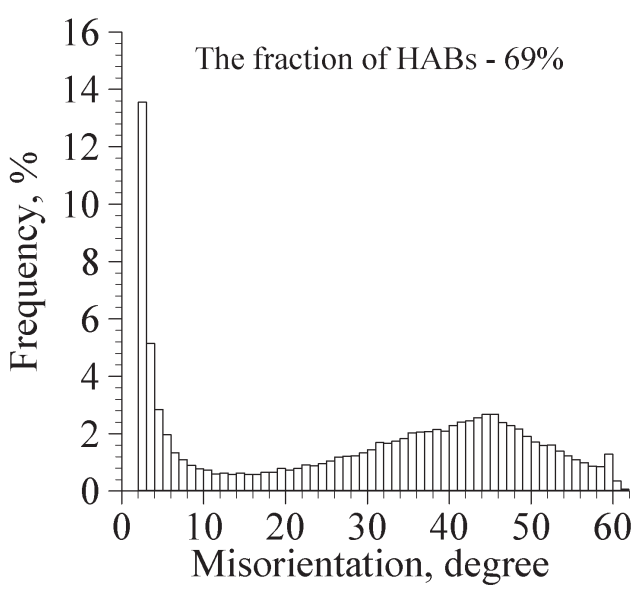

$\mathrm{b}$

Pис. 2. (Color online) Ориентационная EBSD-карта (а) и соответствующий спектр разориентировок границ зерен (b), полученные с центральной части заготовки сплава СДЖС-15, подвергнутой деформационно-термической обработке $\left(T=1175^{\circ} \mathrm{C}, \varepsilon^{\prime} \approx 10^{-2} \mathrm{c}^{-1}\right.$, $e \approx 1.4)$ и старению (НАBs - высокоугловые границы зерен).

Fig. 2. (Color online) EBSD orientation map (a) and corresponding misorientation-angle distribution for grain boundaries (b) obtained for the SDZhS-15 superalloy after thermomechanical treatment $\left(T=1175^{\circ} \mathrm{C}, \varepsilon^{\prime} \approx 10^{-2} \mathrm{~s}^{-1}, e \approx 1.4\right)$ and ageing (HABs - high-angle grain boundaries).

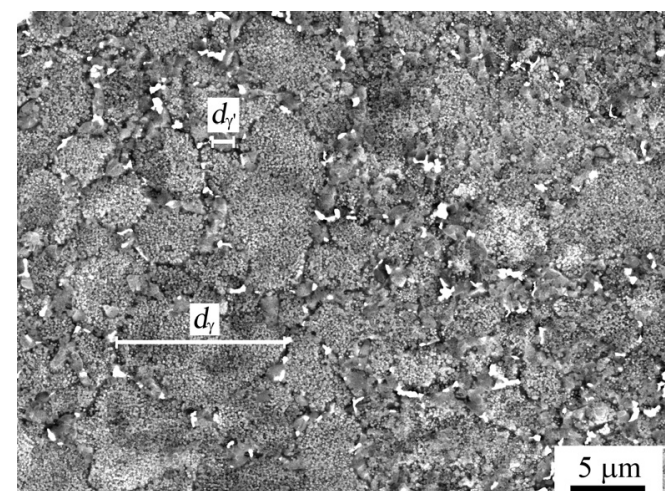

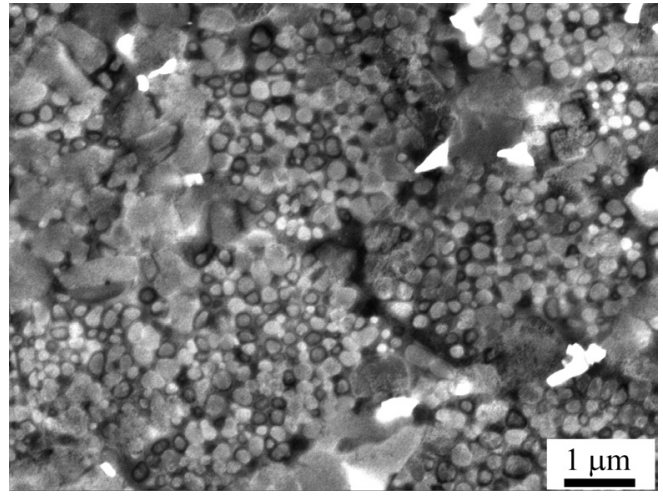

Рис. 3. Электронно-микроскопические изображения микроструктуры сплава СДЖС-15, полученные с центральной части заготовки сплава, подвергнутой деформационно-термической обработке $\left(T=1175^{\circ} \mathrm{C}, \varepsilon^{\prime} \approx 10^{-2} c^{-1}, e \approx 1.4\right)$ и старению: отмечены типичное $\gamma$ зерно и крупное выделение $\gamma^{\prime}$ фазы (a), выделения $\gamma^{\prime}$ фазы (CЭM, BSE) (b).

Fig. 3. Electron microscope images of the SDZhS-15 superalloy obtained from a central part of the thermomechanically treated $\left(T=1175^{\circ} \mathrm{C}\right.$, $\left.\varepsilon^{\prime} \approx 10^{-2} \mathrm{~s}^{-1}, e \approx 1.4\right)$ and aged workpiece: a typical recrystallized $\gamma$ grain and a coarse $\gamma^{\prime}$ precipitate are marked (a), $\gamma^{\prime}$ precipitates (SEM, BSE) (b). 
с исходным состоянием не изменилась. Белые карбидные частицы размером $0.2-3$ мкм располагались в основном вдоль границ $\gamma$ зерен вблизи крупных выделений $\gamma^{\prime}$ фазы.

Литое состояние, подвергнутое ТО, и состояние, полученное после ДТО и старения, были использованы для изготовления образцов и последующего изучения механических свойств сплава.

\section{2. Механические свойства}

В Табл. 1 представлены механические свойства сплава в состояниях после ТО и ДТО. Видно, что свойства, полученные после ДТО, значительно выше свойств сплава, подвергнутого только ТО. Прочность $\left(\sigma_{\mathrm{B}}\right)$ состояния после ДТО оказалась в 1.3-1.5 раза выше прочности состояния после ТО, удлинение в 1.9-3.3 раза выше. Испытания на длительную прочность, выполненные при $650^{\circ} \mathrm{C}$, также выявили различие в свойствах. Образец, подвергнутый ДТО, не разорвался при $650^{\circ} \mathrm{C}$ и нагрузке 1200 МПа после 100-часового испытания, в то время как образец, подвергнутый ТО, не разорвался после 100-часового испытания только при нагрузке $900 \mathrm{MПа.}$

Интересно отметить, что высокие значения длительной прочности были получены для относительно мелкозернистого, рекристаллизованного состояния с преимущественно высокоугловыми границами зерен. По-видимому, высокое легирование тугоплавкими элементами способствовало замедлению диффузии, в том числе зернограничной. Превосходные механические свойства сплава СДЖС-15 после ДТО объясняются повышенным содержанием $\gamma^{\prime}$ фазы, эффективным твердорастворным упрочнением благодаря высокому легированию тугоплавкими элементами, относительно мелкозернистой структурой, полученной в результате ДТО, а также, возможно, присутствием дисперсных карбидов. Выполненная работа показала, что высокое легирование тугоплавкими элементами, включая рений, что характерно для монокристаллических никелевых сплавов, не исключает возможности деформационной обработки. Полученная после ДТО измельченная микроструктура обеспечила не только более высокие механические свойства в сравнении с состоянием сплава после ТО, но и более высокие механические свойства в сравнении со свойствами известных жаропрочных дисковых сплавов на основе никеля.

\section{4. Заключение}

В настоящей работе изучали высоколегированный никелевый сплав СДЖС-15 (Ni-28(Cr, Co)-12.5(Al, Ti, Nb, Ta)-9(Mo, W, Re)-0.17(C, La, Y, Ce, B) (вес. \%)), разработанный в ПАО «ОДК-Сатурн» с помощью компьютерного проектирования и предполагаемый для применения в качестве дискового материала с рабочей температурой $650 \ldots 850^{\circ} \mathrm{C}$. Слиток сплава был подвергнут ТО или ДТО и старению. Установлено следующее:

- после предварительной термической обработки слиток сплава был успешно деформирован в квазиизотермических условиях при температурах немного ниже температуры полного растворения $\gamma^{\prime}$ фазы с использованием специального контейнера. Это обеспечило развитие в сплаве рекристаллизационных процессов и формирование измельченной рекристаллизованной структуры;

- сплав в состоянии после ДТО и старения имел более мелкий размер $\gamma$ зерен, однако размер упрочняющей $\gamma^{\prime}$ фазы в нем оказался крупнее, чем в сплаве, подвергнутом только ТО;

- в состоянии после ДТО и старения сплав показал более высокие механические свойства, чем в литом состоянии после ТО. Прочность сплава после ДТО и старения оказалась на 30-50\% выше

Табл. 1. Механические свойства сплава СДЖС-15 в литом состоянии после термической обработки/в состоянии после деформационно-термической обработки и старения.

Table 1. Mechanical properties of the SDZhS-15 superalloy in the cast and heat treated (HT) condition/in the thermomechanically treated (TMT) and aged condition.

\begin{tabular}{|c|c|c|c|c|}
\hline$T,{ }^{\circ} \mathrm{C}$ & $\sigma_{0.2}, \mathrm{M \Pi a} / \mathrm{MPa}$ & $\sigma_{\text {UTS }}, \mathrm{MПa} / \mathrm{MPa}$ & $\delta, \%$ & $\begin{array}{c}\text { Длительная прочность, МПа } \\
\text { Long-term strength, MPa }\end{array}$ \\
\hline 20 & $1132 / 1355$ & $1255 / 1802$ & $8.5 / 16$ & \\
\hline 650 & $1044 / 1310$ & $1198 / 1558$ & $2.7 / 8.5$ & $T=650^{\circ} \mathrm{C}, \tau=100 \mathrm{~h}$ \\
\hline 750 & $1005 / 1290$ & $1125 / 1462$ & $2.1 / 7$ & >900 (после ТО/after НТ) / >1200 (после ДТО / after ТМТ) \\
\hline 850 & $728 / 1065$ & $733 / 1114$ & $0.9 / 3$ & \\
\hline N18 RT* [9] & 1080 & 1620 & $>20$ & $T=650^{\circ} \mathrm{C}:>1000(\tau=100 \mathrm{~h})$ \\
\hline Alloy $10 \mathrm{RT}[10]$ & 1210 & 1650 & $12-17$ & - \\
\hline AF115 RT [11] & 1190 & 1646 & 20 & $T=650^{\circ} \mathrm{C}: 1030(\tau=100 \mathrm{~h})$ \\
\hline TMW-4 RT [12] & 1210 & 1700 & 9 & - \\
\hline ME3 (Rene104) RT & 1150 & 1580 & $9-18$ & - \\
\hline$[13,14]$ & 1150 & 1650 & 21 & \\
\hline FGH100 RT [15] & 1172 & 1600 & 19 & $T=705^{\circ} \mathrm{C}: 897(\tau=42.8 \mathrm{~h})$ \\
\hline $\mathrm{T}=705^{\circ} \mathrm{C}$ & 1090 & 1345 & 20 & \\
\hline LSHR RT [16] & 1210 & 1700 & $>15$ & - \\
\hline
\end{tabular}

*RT — комнатная температура/room temperature 
прочности литого сплава подвергнутого ТО, при этом выше оказалась также пластичность и длительная прочность при $650^{\circ} \mathrm{C}$;

- механические свойства сплава СДЖС-15 после ДТО и старения оказались выше свойств известных жаропрочных дисковых сплавов на основе никеля.

Благодарности/Aknowledgements. Работа выполнена при поддержке Российского научного фонда (грант РНФ № 18-19-00594). Экспериментальные исследования были выполнены на базе Центра коллективного пользования научным оборудованием ИПСМ РАН./The present work was supported by the Russian Science Foundation (Grant No. № 18-19-00594). The work was performed using the facilities of the shared services center «Structural and Physical-Mechanical Studies of Materials» at the Institute for Metals Superplasticity Problems of Russian Academy of Sciences.

\section{Литература/References}

1. R.C. Reed. The superalloys: Fundamentals and Applications. Cambridge, Cambridge University Press (2006) $372 \mathrm{p}$.

2. M.C. Kushan, S.C. Uzgur, Y. Uzunonat, F. Diltemiz. Recent Advances in Aircraft Technology. Croatia, InTech Rijeka (2012) p. 75 - 96.

3. O.A. Kaibyshev, F.Z. Utyashev. Superplasticity, microstructure refinement and processing of hardto-deform alloys. Moscow, Nauka (2002) 438 p. (in Russian) [O.А. Кайбышев, Ф.3. Утяшев. Сверхпластичность, измельчение микроструктуры и обработка труднодеформируемых сплавов. Москва, Наука (2002) 438 c.]

4. R. A. Hobbs, S. Tin, C. M. F. Rae. Metall. Mater. Trans. A. 36, 2761 (2005). Crossref

5. S. Tin, L. Zhang, R.A. Hobbs, A.-C. Yeh, C.M. F. Rae, B. Broomfield. In: Superalloys 2008 (Ed. by R. C. Reed, P. Caron, T. Gabb, E. Huron, S. Woodare). USA, PA, TMS, Warrendale, Seven Springs (2008) p. 81-90.

6. A. Sato, H. Harada, A.-C. Yeh, K. Kawagishi, T. Kobayashi,
Y. Koizumi, T. Yokokawa, J.-X. Zhang. In: Superalloys 2008 (Ed. by. R. C. Reed, P. Caron, T. Gabb, E. Huron, S. Woodare). USA, PA, TMS, Warrendale, Seven Springs, (2008) p. 131-138.

7. A.V. Logunov, Sh.Kh. Mukhtarov, A.M. Mikhailov, R.A. Gaisin, M. Kh. Mukhametrakhimov, V.M. Imayev. Technology of metals. 6, 3 (2018). (in Russian) [А.В. Логунов, Ш.Х. Мухтаров, А.М. Михайлов, Р.А. Гайсин, М.Х. Мухаметрахимов, В.М. Имаев. Технология металлов. 6, 3 (2018).]

8. Sh.Kh. Mukhtarov, V.M. Imayev, A. V. Logunov, Yu.N. Shmotin, A.M. Mikhailov, R.A. Gaisin, R. V. Shakhov, A. A. Ganeev, R. M. Imayev. Mater. Sci. \& Technol. Submitted for publication (2019).

9. J. Y. Guedou, J. C. Lautridou, Y. Honnorat. In: Superalloys 1992 (Ed. by S. D. Antolovich, R. W. Stusrud, R. A. MacKay, D. L. Anton, T. Khan, R. D. Kissinger, D.L. Klarstrom). USA, PA, TMS, Warrendale, Seven Springs (1992) p. $267-276$

10. D. Rice, P. Kantzos, B. Hann, J. Neumann, R. Helmink. In: Superalloys 2008 (Ed. by R. C. Reed, P. Caron, T. Gabb, E. Huron, S. Woodare). USA, PA, TMS, Warrendale, Seven Springs (2008) p. 139-147.

11. H. Merrick, R.C. Benn, P.R. Bhowal. United States patent US 6,468,368 B1, 22.10.2002.

12. Y.F. Gu, C. Cui, H. Harada, T. Fukuda, D. Ping, A. Mitsuhashi, K. Kato, T. Kobayashi, J. Fujioka. In: Superalloys 2008 (Ed. by R. C. Reed, P. Caron, T. Gabb, E. Huron, S. Woodare). USA, PA, TMS, Warrendale, Seven Springs (2008) p. 53-61.

13. E.S. Huron, K. R. Bain, D.P. Mourer, T.P. Gabb. Superalloys 2008 (Ed. by R. C. Reed, P. Caron, T. Gabb, E. Huron, S. Woodare). USA, PA, TMS, Warrendale, Seven Springs (2008) p. 181-189.

14. Y. Gao, J. S. Stoelken, M. Kumar, R. O. Ritchie. Acta Mater. 55, 3155 (2007). Crossref

15. C. L. Jia, C. C. Ge, Q.Z. Yan. Mater. Sci. \& Eng. A. 659, 287 (2016). Crossref

16. T.P. Gabb, J. Gayda, I. Telesman, P.T. Kantzos. United States patent US 6,974,508 B1, 13.12.2005. 